\title{
Long-term evolution of strongly nonlinear internal solitary waves in a rotating channel
}

\author{
J. C. Sánchez-Garrido ${ }^{1,2}$ and V. Vlasenko ${ }^{3}$ \\ ${ }^{1}$ Grupo de Oceanografía Física. Dpto. Física Aplicada II, Campus de Teatinos, University of Malaga, Malaga, Spain \\ ${ }^{2}$ Grupo de Puertos y Costas, Centro Andaluz de Medio Ambiente, University of Granada, Granada, Spain \\ ${ }^{3}$ School of Earth, Ocean and Enviromental Sciences, Plymouth University, Drake Circus, Plymouth PL8 4AA, UK
}

Received: 31 May 2009 - Accepted: 4 September 2009 - Published: 25 September 2009

\begin{abstract}
The evolution of internal solitary waves (ISWs) propagating in a rotating channel is studied numerically in the framework of a fully-nonlinear, nonhydrostatic numerical model. The aim of modelling efforts was the investigation of strongly-nonlinear effects, which are beyond the applicability of weakly nonlinear theories. Results reveal that small-amplitude waves and sufficiently strong ISWs evolve differently under the action of rotation. At the first stage of evolution an initially two-dimensional ISW transforms according to the scenario described by the rotation modified Kadomtsev-Petviashvili equation, namely, it starts to evolve into a Kelvin wave (with exponential decay of the wave amplitude across the channel) with front curved backwards. This transition is accompanied by a permanent radiation of secondary Poincaré waves attached to the leading wave. However, in a strongly-nonlinear limit not all the energy is transmitted to secondary radiated waves. Part of it returns to the leading wave as a result of nonlinear interactions with secondary Kelvin waves generated in the course of time. This leads to the formation of a slowly attenuating quasi-stationary system of leading Kelvin waves, capable of propagating for several hundreds hours as a localized wave packet.
\end{abstract}

\section{Introduction}

Oceanic internal solitary waves (ISWs) are typically the most energetic vertical motions of the World Ocean. In some places, their amplitude reach $100 \mathrm{~m}$ or even more (Wesson and Gregg, 1988; Yang et al., 2004). These waves produce shear currents and turbulence (Garrett, 2003) that mix wa-

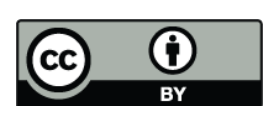

Correspondence to:

J. C. Sánchez Garrido

(jcsanchez@ctima.uma.es) ter and supply nutrients from the abyss to the surface photic layer and enhance biological productivity (Briscoe, 1984).

There are a number of mechanisms of internal wave generation. It is commonly believed that one of the principal sources of oceanic internal waves is the interaction of barotropic tides with prominent bathymetric features. There are many parameters that control their generation, subsequent evolution and ultimate dissipation, such as tidal excursion, bottom topography, and background stratification. The rotation of the Earth plays a fundamental role in the dynamics of baroclinic tidal waves (Vlasenko et al., 2005), both in generation and their disintegration into packets of ISWs. Gerkema and Zimmerman (1995) and Gerkema (1996) were probably the first to find that in weakly nonlinear nonhydrostatic media, sufficiently strong rotation suppresses disintegration of progressive baroclinic tidal wave into packets of short ISWs. This theory was recently revised by Helfrich and Grimshaw (2008), who considered how the balance between hydrostatic and nonhydrostatic parts of the long-wave solution influences its disintegration into a packet of ISWs. However, after its generation these short-scale ISWs are mostly unaffected by rotation due to the fact that nonhydrostatic dispersion exceeds substantially the rotational dispersion. A noticeable effect can be expected when these two dispersions are comparable.

In a very general theoretical context, the influence of rotation on ISWs was considered in a number of papers where the Ostrovsky's equation (Ostrovsky, 1978), which incorporates weak nonlinearity and rotational dispersion, was subjected to an asymptotic analysis. One of the conclusions was that internal waves of permanent form cannot exist in rotating systems (Leonov, 1981).

Under weak rotation, when a KdV solitary wave is used as the initial condition of the Ostrovsky equation, it decays due to radiation of inertial gravity waves (Grimshaw et al., 1998). Details of the dynamics were described by Gilman et al. (1996): radiated long inertial waves eventually steepen to

Published by Copernicus Publications on behalf of the European Geosciences Union and the American Geophysical Union. 
produce a new solitary wave, which in turn fades out repeating the process of wave-radiation and further formation of a new solitary wave. The long-term behaviour of this recurrent mechanism was recently investigated in detail by Helfrich (2007). It was found that in a strongly-nonlinear case not all the energy of the initial wave radiates with secondary waves. A certain part of initial energy (up to a half in some cases) can accumulate in a packet of amplitude modulated solitary waves that propagate through a long wave envelope. The group velocity of the envelope is less than the phase speed of every individual solitary wave. As a result, solitary waves emerge in the tail, propagate forward through the envelope growing in amplitude until the middle of the packet, to finally attenuate and decay at the leading edge.

Rotational effects develop even more dramatically in constrained systems like straits and channels. The influence of lateral boundaries leads to the formation of radiated Poincaré waves and a trapped Kelvin wave. This was observed for the first time in a laboratory tank by Maxworthy (1983), who conducted experiments with stratified fluids and pointed out that rotation causes the wave crest of the solitary wave to be curved backwards. The findings were later confirmed by Renouard et al. (1987), who also noticed that the leading Kelvin wave is accompanied by a train of small amplitude waves trailing the main disturbance.

The detailed analysis of the evolution process of ISWs in a rotating channel was performed in a number of theoretical works (Grimshaw, 1985; Katsis and Akylas, 1987; Grimshaw, 1988; Melville et al., 1989; Grimshaw and Tang, 1990; Akylas, 1991). The analysis is normally based on the Rotation Modified Kadomtsev-Petviashvili (RMKP) equation for the wave amplitude $A(x, y, t)$, where $x, y$ and $t$ are along-channel, across-channel coordinate and time, respectively. With the appropriate boundary conditions at the lateral boundaries $(y=0, l)$ the problem on evolution of weakly nonlinear internal waves in a rotating channel reads:

$$
\begin{aligned}
& \left(A_{t}+\nu A A_{x}+\lambda A_{x x x}\right)_{x}+\frac{1}{2} c_{0}\left(A_{y y}-\gamma^{2} A\right)=0, \\
& A_{y}+\gamma A=0, \quad y=0, l .
\end{aligned}
$$

Here $c_{0}$ is the linear phase speed and $\gamma=R_{0}^{-1}$ is the inverse internal Rossby radius which provides a measure of rotation. The parameters $v$ and $\lambda$ are coefficients of nonlinearity and dispersion respectively, both dependent of the associated vertical mode $g_{0}$ calculated from the eigen-value problem:

$$
\begin{aligned}
& \frac{d^{2} g_{j}}{d z^{2}}+\frac{N^{2}}{c_{j}^{2}} g_{j}=0, j=0,1, \ldots \\
& g_{j}(-H)=g_{j}(0)=0, j=0,1, \ldots
\end{aligned}
$$

Here $N(z)$ is the buoyancy frequency and $H$ the water depth.

Grimshaw (1985) was the first to formulate the problem (1)-(2). It was used for the study of propagation of weakly nonlinear internal waves in a continuously stratified rotating shallow-water channel in the case when the rotation as well as nonlinearity, three-dimensional effects and dispersion are weak and of the same order. Conditions (2) constrain the choice of initial solitary form satisfying Eq. (1) by waves in which their mass in planes parallel to lateral walls varies exponentially across the channel. An attempt to find a solitarywave solution of the RMKP equation in the form suggested by Maxworthy (1983) (with straight or curved wave front) was not successful because of the boundary conditions (2). This problem was studied numerically for two-layer fluid by Katsis and Akylas (1987), who noticed that the initially straight-crest SIW transforms into a wave with exponential decrease of amplitude (characteristic of a Kelvin wave) with wave crest curved backwards. The wave suffered remarkable attenuation due to the radiation of secondary perturbances. Similar results were obtained by Grimshaw and Tang (1990) for continuously stratified fluids.

The system of waves satisfying both the RMKP Eq. (1) and boundary conditions (2) was suggested by Grimshaw and Tang (1990) in the form:

$A=\sum_{n=0}^{\infty} A_{n}(x, t) \phi_{n}(y)$,

where $\phi_{0}=e^{-\gamma y} ; \phi_{n}=\cos \left(\frac{n \pi y}{l}\right)-\frac{\gamma l}{n \pi} \sin \left(\frac{n \pi y}{l}\right), n=1,2 \ldots$ are the complete set of orthogonal functions in which $n=0$ represents the trapped Kelvin mode, and $n \geq 1$ the radiated Poincaré modes. The following equations for the wave amplitude of each mode was found:

$$
\begin{aligned}
& A_{0 t}+\lambda A_{0 x x x}+\frac{1}{2} v\left\{\sum_{p=0}^{\infty} \sum_{q=0}^{\infty} C_{0 p q}\left(A_{p} A_{q}\right)_{x}\right\}=0 \\
& \left(A_{n t}+\lambda A_{n x x x}\right)_{x}+b_{n}^{2} A_{n}-\frac{1}{2} v\left\{\sum_{p=0}^{\infty} \sum_{q=0}^{\infty} C_{n p q}\left(A_{p} A_{q}\right)_{x x}\right\}=0
\end{aligned}
$$

Here $C_{n p q}=\frac{\int_{l}^{0} \phi_{n} \phi_{p} \phi_{q} d y}{\int_{l}^{0} \phi_{n}^{2} d y}, b_{n}^{2}=\frac{1}{2} c_{0}\left(\gamma^{2}+\frac{n^{2} \pi^{2}}{l^{2}}\right)$. Note that Eqs. (6) and (7) are coupled in the nonlinear terms, and, as pointed out by Grimshaw and Tang (1990), this is the ultimate cause that inhibits the existence of a stationary solution. To illustrate the mechanism, let us suppose the inexistence of Poincaré modes at $t=0\left(A_{i}=0, i=1, \ldots, n\right.$, for $\left.t=0\right)$. In this case (6) admits the solitary wave solution which, together with the transversal Kelvin mode $\phi_{0}$, forms a Kelvin solitary wave taken as initial condition. Following Eq. (7), the Kelvin mode itself will excite the Poincaré modes, which in turn subtract energy from the initial wave and are radiated backwards due to their lower phase speed.

If one takes a straight-crest wave across the channel for the initial wave as Renouard et al. (1987) did, it starts to evolve as discussed above transforming into exponentially decreased across the channel wave with curved backwards front. Even 
though the wave profile consistent with the boundary condition (2) is taken as the initial wave, it also starts to evolve according to the scenario described by Katsis and Akylas (1987) and Grimshaw and Tang (1990). One of the conclusions of these papers was that the exponential decrease of the wave amplitude across the channel is not a crucial condition for the wave evolution.

Thus, we used this result as a justification of our approach to take a straight-crest ISW as initial condition for the investigation of its long-term evolution. The new element introduced in this study is that our analysis is not restricted to only weakly-nonlinear waves. The initial amplitude can be as large as $100 \mathrm{~m}$ or more for oceanic-scale waves (as observed in many places), which demands the application of a fully-nonlinear approach.

This paper investigates numerically the propagation of ISWs in a rotating rectangular channel focusing on long-term effects of rotation on their structure and evolution. It is organized as follows: Sect. 2 describes the numerical model and its initialisation. In Sect. 3 the reference numerical experiment is discussed. Sensitivity runs are examined in Sect. 4. Finally, Sect. 5 contains discussions and conclusions.

\section{Model description and initialisation}

Some results on modelling of ISWs propagating in the Strait of Gibraltar have been recently reported by Vlasenko et al. (2009). One conclusion was that this strait is too short for weak rotational dispersion to introduce any substantial corrections into the wave field. The rotational effects in the Strait of Gibraltar typically account for only about $10 \%$ of wave amplitude. The purpose of this paper is to study the situations of long-term evolution in order to clarify the ultimate stage of propagation of strongly nonlinear ISWs under the action of rotation. In doing so, we simplify the bottom topography considering only rectangular straight channels. This circumstance excludes any effects related to the scattering of internal waves by variable bottom topography. Hence, all changes in the wave structure can be attributed to the influence of rotation. Specifically, in our analysis the parameters of the channel and propagating waves were taken close to those observed in the Strait of Gibraltar: the width and depth were $17 \mathrm{~km}$ and $600 \mathrm{~m}$ respectively, the vertical fluid stratification as presented in Fig. 1, and amplitudes of incoming ISWs from 10 to $90 \mathrm{~m}$.

The Massachusetts Institute of Technology general circulation model (MITgcm), which is a fully non-linear nonhydrostatic hydrodynamic model, was used in our study. A detailed description of the model can be found in the paper by Marshall et al. (1997). At first stage the model was used for the preparation of the initial fields. It was initialized by the first-mode solitary wave of depression obtained from the Korteveg-de Vries (KdV) equation. This wave represents a stationary weakly non-linear solution of the $\mathrm{KdV}$ equation. It

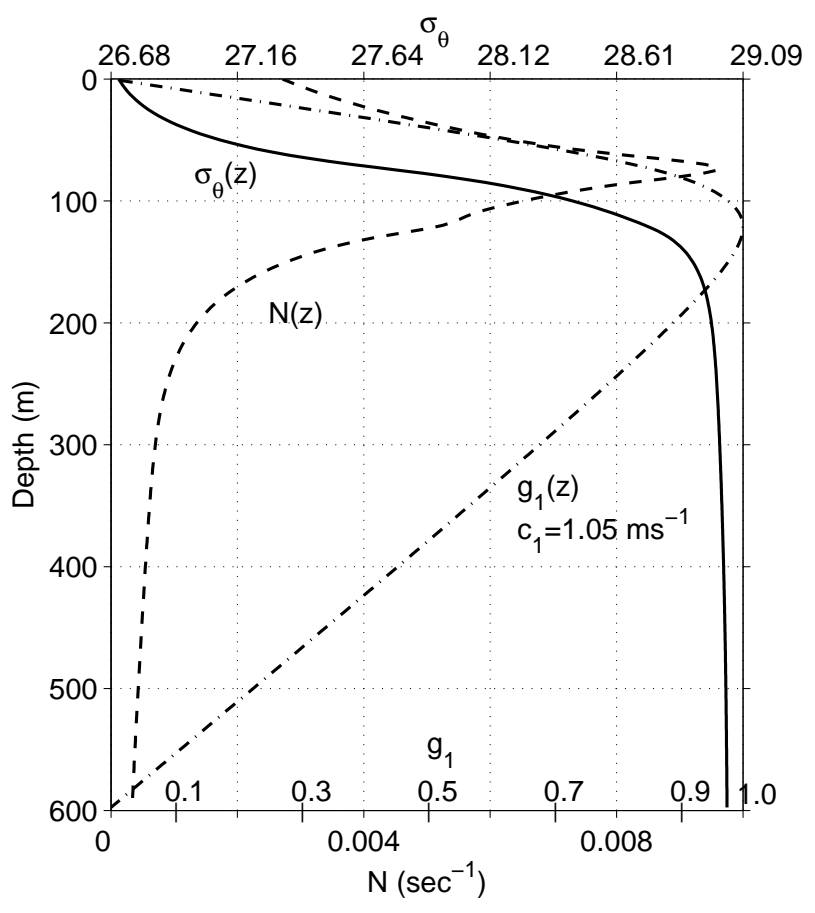

Fig. 1. Solid line: density profile used in the numerical experiments. Dashed line: associated buoyancy frequency. Dashed-point line: Normalizad vertical wave displacement of the first baroclinic mode.

does not however satisfy the fully nonlinear non-hydrostatic equations in the case of large amplitude waves considered here. Hence, being inserted into the numerical model the wave starts to evolve (even without rotation) eventually transforming into a new steady-state wave in which strong nonlinearity is in balance with all other effects. It takes about 20-30 wavelengths for the leading wave to evolve into an isolated stable ISW well detached from the secondary wave tail. This leading ISW is taken hereafter as initial field for our numerical experiments.

The computational domain included a rectangular grid with resolution of $\Delta x=50 \mathrm{~m}, \Delta y=900 \mathrm{~m}$, along and crosschannel respectively, and vertical step $\Delta z=7.5 \mathrm{~m}$ in the upper $300 \mathrm{~m}$ layer and $50 \mathrm{~m}$ below it. This configuration turned out to be quite realistic to capture all the details of the three-dimensional evolution of ISWs. All other model parameters were as follows: vertical turbulent viscosity and diffusivity coefficients were $\nu_{V}=10^{-3} \mathrm{~m}^{2} \mathrm{~s}^{-1}$ and $\kappa_{V}=10^{-5} \mathrm{~m}^{2} \mathrm{~s}^{-1}$ respectively, whereas horizontal coefficients were $v_{H}=\kappa_{H}=10^{-2} \mathrm{~m}^{2} \mathrm{~s}^{-1}$.

At the eastern boundary of the domain we set zero values for all wave disturbances provided that the wave do not reach this boundary. At the western boundary, the radiating type Orlanski open boundary conditions were used. Regarding the latter, we note that in addition the western boundary is taken far enough to prevent any reflected unphysical signals. At the closed boundaries, the non-slip condition is imposed. 


\section{Reference experiment}

In this section we analyse the reference experiment (RE), in which the evolution of a $83 \mathrm{~m}$-amplitude ISW in a rectangular channel of $l=17 \mathrm{~km}$ width is investigated. Note that the latitude in the RE is equal to $60^{\circ}$, which is higher than the latitude of the Strait of Gibraltar $\left(36^{\circ}\right)$. Higher latitude was taken in order to enhance the effect of rotation; weaker rotation is considered later.

Figure 2 shows a plan view of the wave field at several stages of the ISW evolution. The free surface perturbations produced by the internal waves are used as a tracer of wave fronts. This method of visualization is possible because internal waves of depression produce small-scale elevations at the free surface (a few centimetres, in fact), while internal waves of elevation produce similar small-scale depressions (elevation and depression are illustrated in dark and light colour, respectively). For instance, Fig. 2a shows the initial plane ISW of depression of $83 \mathrm{~m}$ amplitude.

The wave pattern presented in Fig. $2 \mathrm{a}-\mathrm{c}$ reveals that the above discussed effects related to the influence of rotation on the propagation of a weakly nonlinear ISW, i.e. formation of a leading Kelvin wave with strong attenuation due to the radiation of secondary Poincaré waves, take place in a stronglynonlinear case as well. After $14.4 \mathrm{~h}$ of evolution (Fig. 2b) the initially plane ISW loses its original two-dimensional structure and starts to radiate secondary Poincaré waves. Due to this energy leakage its amplitude rapidly decreases from $83 \mathrm{~m}$ to about $60-68 \mathrm{~m}$ during this early stage of evolution (see black solid numbers in Fig. 2, representing the wave amplitude at the positions where they are located). In addition to that, the leading wave gradually transforms into a Kelvin wave with its front curved backwards (Fig. 2c-f), as it was discussed in the aforementioned papers on the dynamics of weakly nonlinear waves.

The similarity between the evolution of weak and strong waves does not hold however for a long period of time. At the latest stages of evolution, weakly nonlinear and strongly nonlinear waves behave differently. In contrast to weak ISWs, which slowly attenuate and gradually vanish transforming their energy to the radiated Poincaré waves, the large-amplitude ISW produces also a fairly pronounced wave packet of secondary Kelvin waves attached behind (see Fig. $2 \mathrm{~g}$ ). This is quite a new result which has not been reported yet.

It is assumed that the formation of a packet of secondary Kelvin waves is possible because of the large quantity of energy accumulated in the initial ISW. As a result, not all the energy of the initial wave is radiated with the Poincaré waves, but part of it remains allocated in the leading wave packet. A similar result on the formation of a localized secondary wave packet from the initial large ISW propagating in infinite rotating basin has been reported recently by Helfrich (2007). The process of self-organisation of secondary Poincaré waves into a packet of Kelvin waves is shown in
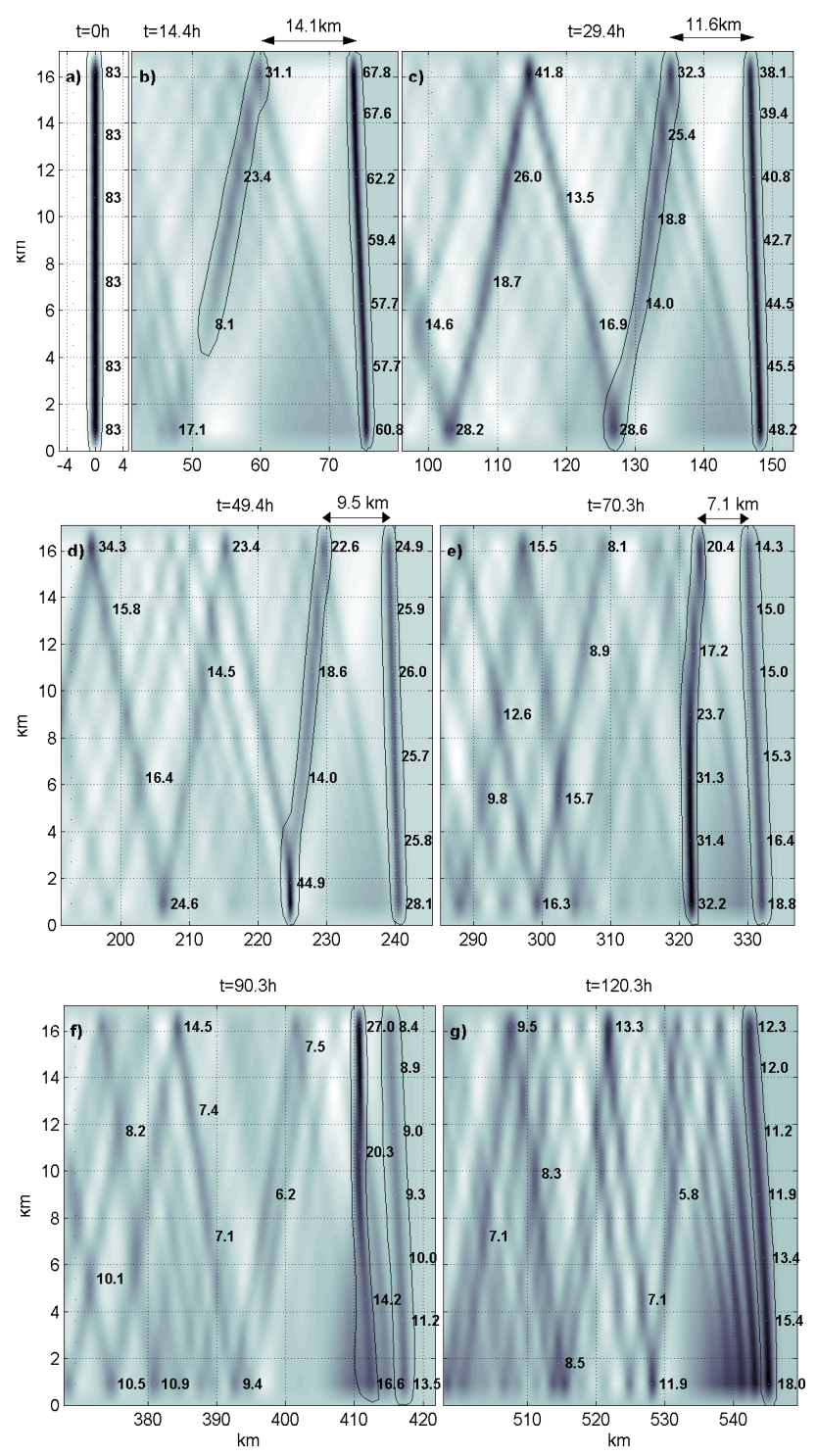

Fig. 2. Sea surface topography produced by a ISW of depression at different stages of evolution. Initial amplitudes of propagating wave was $83 \mathrm{~m}$. Surface elevations and depressions are shown by black and white colours, respectively. The black numbers on the wave fronts represent the wave amplitude (in metres) at their particular positions. The contours containing the leading and secondary waves indicate the volume where total energy is calculated.

Fig. 2b-g. At the first stage, the radiated waves are relatively weak with their crests tilted backwards. The strongest waves experience multiple reflections from the channel boundaries. It is interesting that the wave amplitude at the point of reflection is much larger than in the middle of the channel. This is a manifestation of a nonlinear effect of wave interaction when the obliquely propagating ISWs, incident and reflected from the wall, generate the Mach stem in the place of their crossing, i.e. near the wall in the present case. Several of 
those fragments are seen in Fig. 2b, c and d (compare wave amplitudes in the place of reflection with the central parts of the channel).

In the course of time, the Mach stems gradually transform into a system of secondary Kelvin waves. Analysis of their position gives an amazing result that Mach-stem fragments overcome the leading wave even though they are weaker (the distance between the leading and second wave decreases from $14.1 \mathrm{~km}$ at $t=14.4 \mathrm{~h}$ to $9.5 \mathrm{~km}$ at $t=49.4 \mathrm{~h}$ and $7.1 \mathrm{~km}$ at $t=70.3 \mathrm{~h}$ ). This effect cannot be explained in terms of traditional nonlinear dispersion because the amplitudes of the secondary wave are substantially smaller until $t=40 \mathrm{~h}$, however the distance between the leading ISW and secondary waves decreases over time. During the evolution, the second wave gets stronger adsorbing the energy from the leading wave and gradually approaching it (compare panels d, e and f). Finally, after $120 \mathrm{~h}$ the initial large-amplitude ISW transforms into a well rank-ordered packet of internal Kelvin waves with their fronts curved backwards. This structure is followed by a wave tail with chaotic spatial distribution of amplitudes (see Fig. 2d).

An explanation of this transformation (fast wave tail propagation, in particular) can be found in terms of permanent energy radiation. In fact, the system of secondary waves propagating behind the leading ISW is not a quasi-stationary structure, but a system where new born waves are permanently generated. In the course of evolution, the leading ISW continuously loses its energy decreasing in amplitude and decelerating due to the nonlinear dispersion. As a result of the wave weakening, the new-born radiated wave is generated closer and closer to it. This effect has an analogy with the structure of a shock wave produced by a propagating supersonic object in which the Mach cone gets wider when the speed of the object decreases.

To confirm the hypothesis that the structure of the attached wave tail remains quasi-stable only due to the fact of a permanent transfer of energy from the leading wave to the tail, an additional numerical experiment has been conducted. The initial condition for this experiment was the wave fields taken from the RE at $t=10 \mathrm{~h}$ when both the leading Kelvin ISW and the attached radiated Poincaré waves are well developed. They are shown in Fig. 3a1. In order to prevent the effect of the continuous energy transfer to the wave tail, the wave field was "truncated" by removing the leading ISW. The resulting field is presented in Fig. 3 (compare panels a1 and a2). The evolution of the truncated wave system is shown in Fig. 3 by panels $\mathrm{b} 2$ and $\mathrm{c} 2$, whereas panels $\mathrm{b} 1$ and $\mathrm{c} 1$ show the wave field corresponding to the RE (without cutting of the leading ISW) at the same times.

The "far field" of the radiated secondary Poincaré waves in the RE and the "truncated" experiment (to the left of $x=120 \mathrm{~km}$ in panels $3 \mathrm{c} 1$ and $3 \mathrm{c} 2$ ) coincides almost perfectly. The "far field" does not feel the truncation of the leading wave during the first $20 \mathrm{~h}$ of evolution at all (probably because of the large distance). On the other hand, the
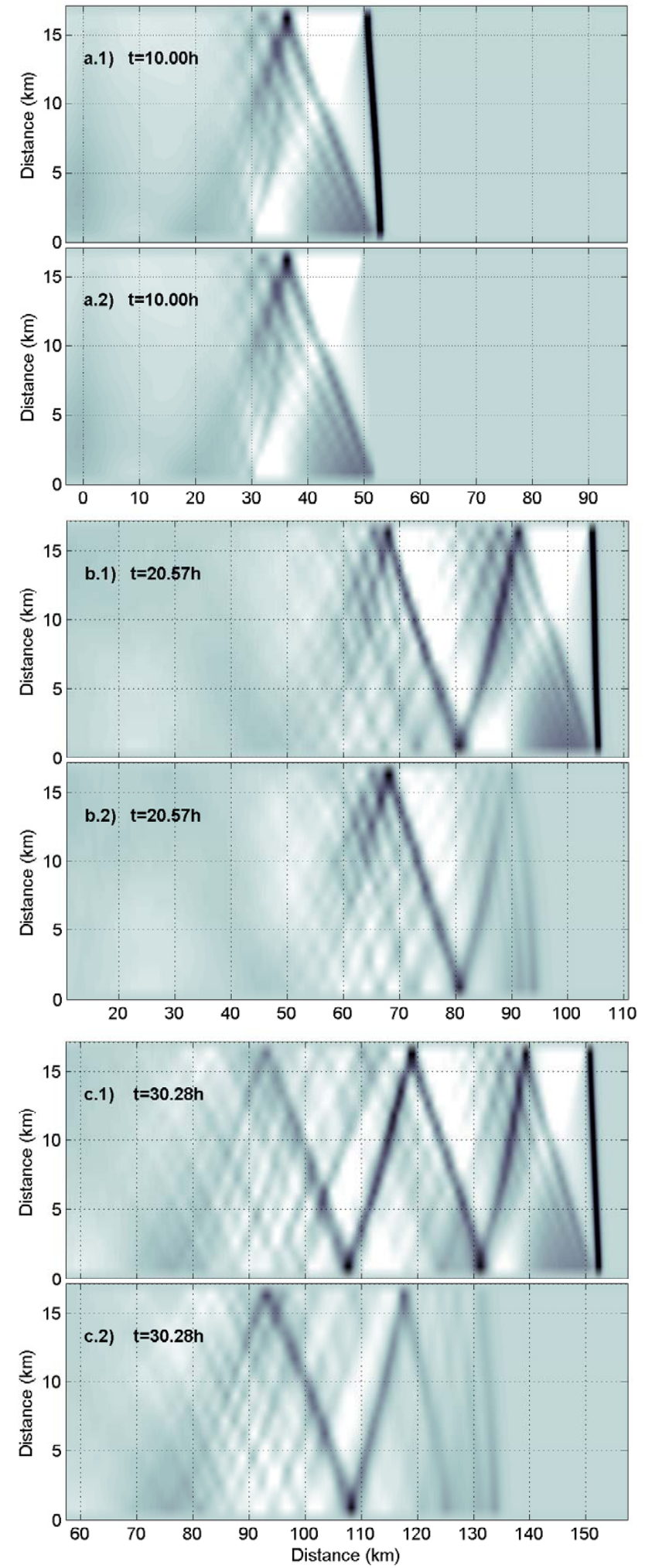

Fig. 3. Evolution of ISW in the reference experiment (panels a1, b1 and c1, see also Fig. 2) and the "truncated" experiment (panels a2, $\mathrm{b} 2$ and c2) when the leading ISW was removed from the wave field at $t=10 \mathrm{~h}$ (compare panels a 1 and $\mathrm{a} 2$ ). 


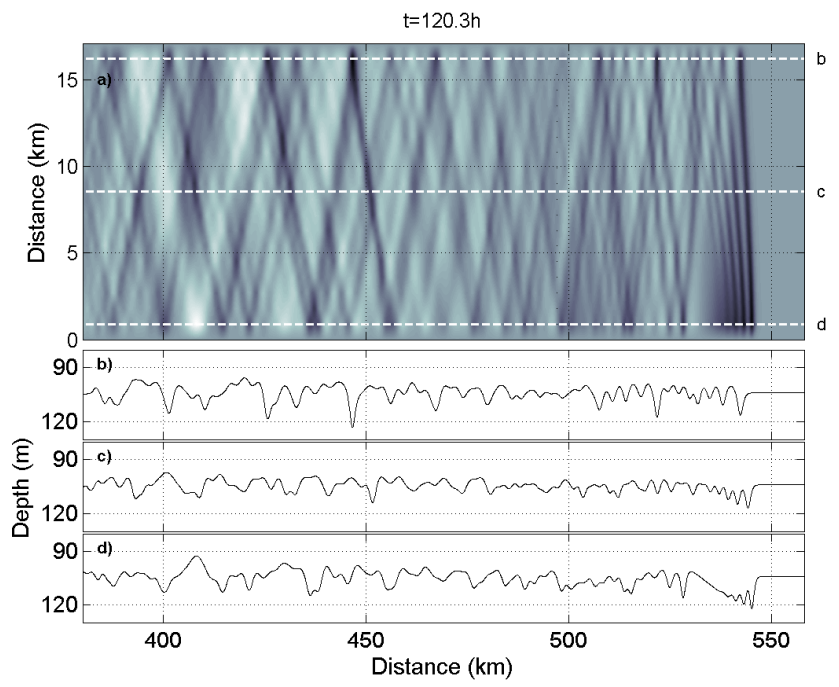

Fig. 4. (a) Same as in Fig. $1 \mathrm{~g}$ but covering a larger area. Panels (b), (c), (d) show the isopycnal $\sigma_{\theta}=28.5$ along the cross sections indicated by white-dashed lines in panel (a).

"near-field" (between $x=135$ and $150 \mathrm{~km}$ in the same panels) in both experiments is completely different. It is empty in the "truncation experiment" (no internal wave signal is observed in this zone), a fact which allows to conclude that the "nearfield" is really generated exclusively by the leading ISW, but not by a superposition of overcoming old radiated waves and new-born Poincaré waves.

Thus, the basic outcome from this analysis is that the reconstruction and discussed "compression" of the leading wave field at the early stage of the experiment can be attributed to the high rate of energy loss of the leading ISW. On the other hand, at the latest stages (when $t>50 \mathrm{~h}$ ) the wave amplitude of secondary waves gets even larger than that in the leading ISW (see Fig. 2e-f). This is the reason why the nonlinear effects due to the amplitude dispersion make an additional compression of the packet possible. The nonlinear collision of several incidentally propagating waves and energy transfer from overcoming stronger rear waves to weaker leading waves (Fig. 2f) result in the formation of a well rankordered packet of Kelvin waves (see Fig. 2g).

Let us consider some more spatial characteristics of the "far-field" of the radiated waves. The large fragment of the model domain is shown in Fig. 4. Panel 4a represents the plan-view of the wave field (surface elevation, as in Figs. 2 and 3) which can be characterised as a superposition of several mostly regular systems of obliquely propagating internal waves generated by multiple reflections from the boundaries. This superposition leads to a less regular spatial distribution of wave amplitude along the channel, as it is seen in Fig. 4b$\mathrm{d}$ where the profile of isopycnal $\sigma_{\theta}=28.5$ along the northern, central and southern sections of the channel is presented. It is clear that only the leading wave packet of Kelvin waves

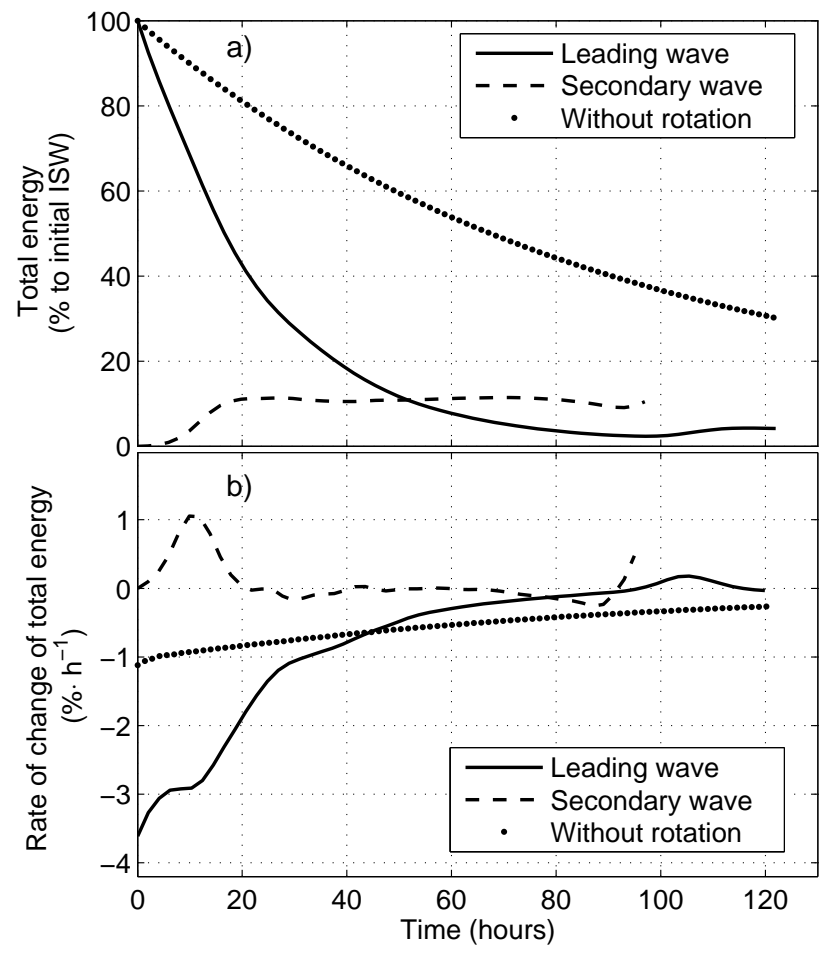

Fig. 5. (a) The total wave energy (kinetic and available potential) of the leading ISW normalized by its initial energy (solid line) and the energy of the secondary wave (dashed line) generated in the RE (see Fig. 2). Dotted line represents the evolution of total energy of the same initial wave (Fig. 3a) propagating in a nonrotating channel. (b) Rate of change of the total energy $\partial E / \partial t$ over time.

is well rank-ordered whereas the amplitude of the radiated wave tail is nearly randomly distributed (locally they are even larger than the leading waves).

In order to evaluate the intensity of the energy leakage due to radiation of Poincaré waves and to compare this loss with the effect of viscosity, another numerical experiment was undertaken. All conditions were kept the same as in the RE except for the rotation, which was removed from the analysis. As long as the nonlinearity and dispersion in the initial ISW are in balance (see Sect. 2), all changes in its energy (amplitude) can be attributed only to the viscous damping. Figure 5a shows the evolution of the total energy (kinetic and available potential) for both cases, with and without rotation. The influence of the viscosity on the wave damping is substantially weaker than from the rotation. At all stages the total energy of the leading ISW is several times larger in the case without rotation. This is the result of the wave radiation: during the first $20 \mathrm{~h}$, the total energy decreases several times faster due to this effect rather than due to the viscous damping (see Fig. 5b, where the rate of change of total energy is presented).

It is interesting to remark that the energy of secondary waves formed within the first $20 \mathrm{~h}$ (dashed lines in Fig. 5) 

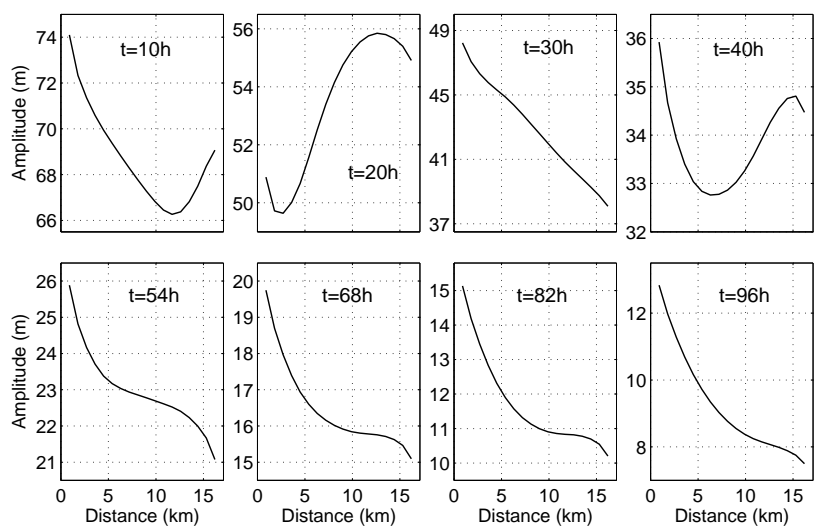

Fig. 6. Across channel variations of amplitude of the leading wave at different stages of its evolution.

is practically conserved over the whole period of evolution. The conservation of energy is established because of the equilibrium between the energy leakage (viscosity, radiation) and energy supply from the leading wave. Another important outcome is that the leading ISW does not only lose energy, but can also gain some part of it from the wave tail after $100 \mathrm{~h}$ (see Fig. 5) as a consequence of the nonlinear wave-wave collision, as discussed above (see Fig. 2f).

The high rate of energy loss of the leading wave during the first part of the experiment is possible because of the radiation of energetic Poincaré modes. In fact, the classification of the leading SIW as a Kelvin wave is only correct after passing a transition time which is necessary to radiate such higher order transversal modes. Evidence of Poincaré waves is observed in Fig. 6, where the transverse distribution of the amplitude of the leading wave is shown at different times. The influence of large-amplitude Poincaré modes over the first $40 \mathrm{~h}$ of the experiment is clear from the transverse wave amplitude oscillations (compare panels in the upper row). With the course of time these waves are radiated and a well developed exponential decrease of the wave amplitude across the channel, peculiar to the Kelvin mode, is established (second row in Fig. 6). Note that despite the shape of this curve is fairly stable and close to the exponential one, the amplitude of the leading wave gradually decreases due to a permanent radiation damping.

Another evidence of wave attenuation and higher mode existence is shown in Fig. 7, which shows the temporal variations of the wave amplitude at the northern, central and southern section of the channel (see Fig. 4a). The wave amplitude of the experiment without rotation is included for comparison. Unsteady transverse oscillations of amplitudes last for about $40 \mathrm{~h}$. The temporal growth of amplitude after $120 \mathrm{~h}$ is just a manifestation of the nonlinear collision of two waves discussed above (see also Fig. $2 \mathrm{f}$ and g).

Once examined, the evolution mechanism rises the question of whether the leading packet of Kelvin solitary waves

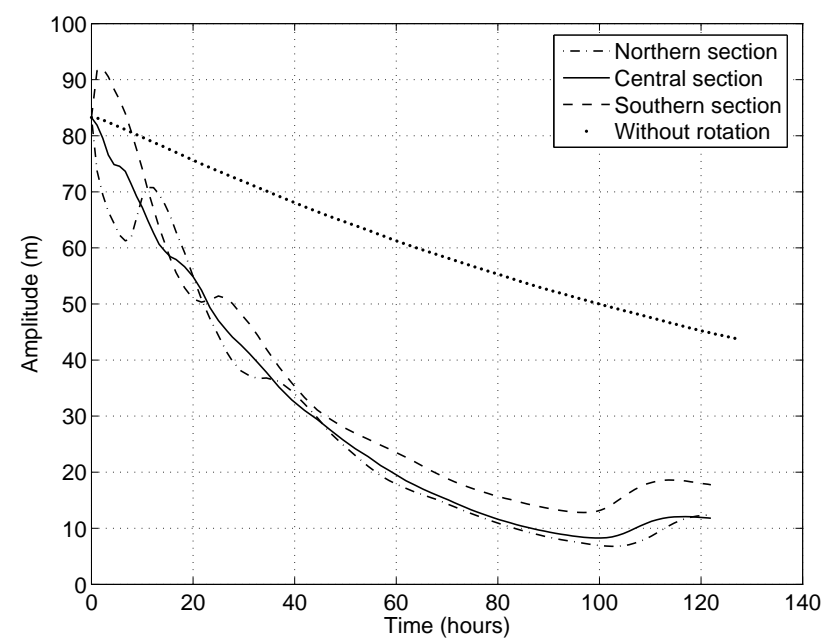

Fig. 7. The temporal dependence of amplitude along the northern (dashed-dotted line), central (solid line) and sothern (dashed line) sections of the channel (Fig. 4a). The variations of amplitude for the non-rotation experiment is depicted in dotted line.

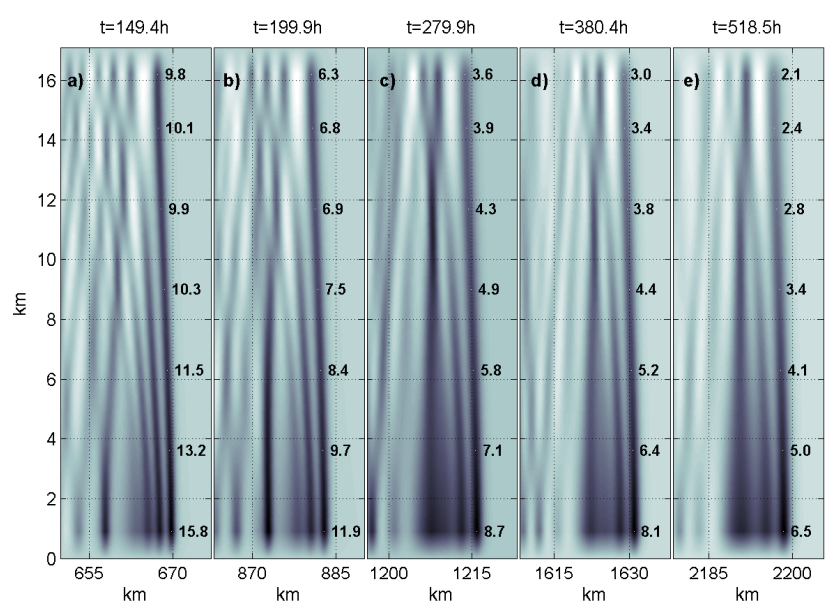

Fig. 8. Extension of the wave field presented in Fig. 2.

presented in Fig. $2 \mathrm{~g}$ is a stable or quasi-stable structure, or it is under permanent reconstruction. In other words, we should answer the question whether the energy radiation is still remarkable at the latest stages of evolution or the wave pattern preserves (at least partly) its quasi-permanent form. Figure 8 shows an extension of the RE up to $518 \mathrm{~h}$. Panels $8 \mathrm{a}-\mathrm{b}$ reveals that the first scenario takes place: a secondary disturbance is formed in the far-field of the packet, overtakes it and interacts with the leading wave packet (Fig. 8c) according to the process described above. As a result of this interaction, the leading wave packet adsorbs the incoming wave, and a new structure containing three internal waves emerges. The latter, in fact, has a very similar structure as the initial one but at a lower energy level. During the last 238 h (Fig. 8c-e) 

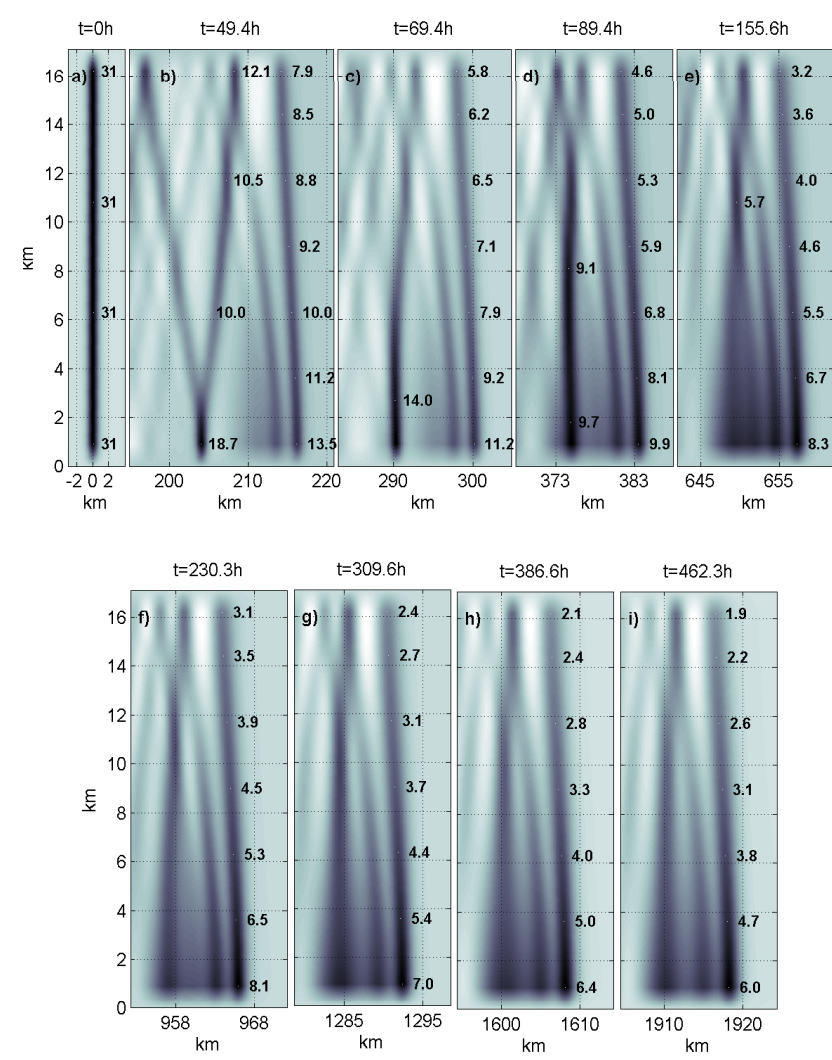

Fig. 9. Same as Fig. 2 but for an initial 31-metre amplitude ISW.

the amplitudes of the leading wave is reduced by about $40 \%$. Taking into account that in the RE the amplitude decrease due to viscosity during the first $130 \mathrm{~h}$ (Fig. 7) comprises a similar value, one can conclude that the basic reason for the wave damping at the latest stages of evolution is the viscous dissipation rather than the radiation of secondary waves.

\section{Sensitivity runs}

\subsection{Sensitivity to the initial wave-amplitude}

In this section sensitivity of the model output to the amplitude of the initial solitary wave is analysed. The evolution of an ISW with $31 \mathrm{~m}$ amplitude (about three times smaller than that in the RE) is presented in Fig. 9. The evolution in this case is quite similar to the RE (Fig. 2), namely, fast attenuation of the leading wave accompanied by the generation of radiated Poincaré waves, formation of Mach stems near the wall (Fig. 9b) with subsequent evolution into a secondary Kelvin wave that overtakes the leading disturbance, and the final formation of a wave train (see Fig. 9c-e).

Nevertheless some details are certainly different in the two numerical experiments. The secondary Kelvin wave is developed earlier in the RE (compare Fig. 2 and Fig. 9), and there is no further formation of a third Kelvin wave as it occurred

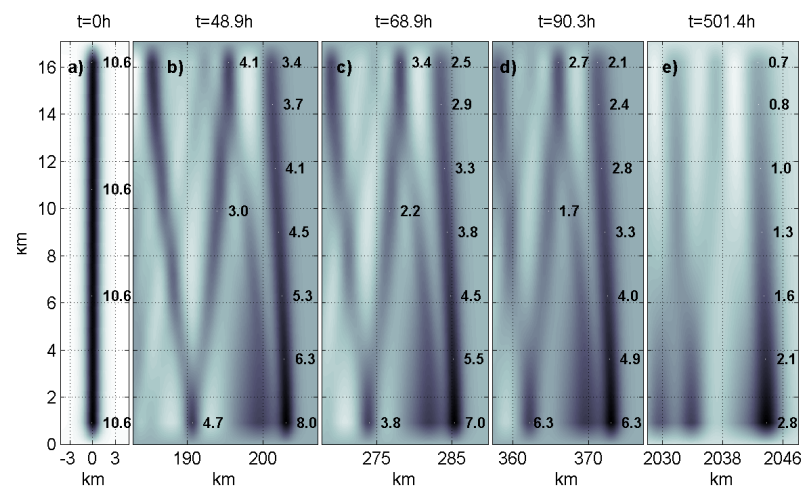

Fig. 10. Same as Fig. 2 but for incoming 10-m amplitude ISW.

in the RE (Fig. 8a). After the interaction with the overtaking secondary wave (Fig. 9e), all transition processes in the wave packet become slower, and the latter conserves quite a stationary form for a long time $(300 \mathrm{~h}$ at least) with weak energy leakage. It is interesting that despite the fact that the initial ISW was remarkably smaller than in the RE and, as a result, its evolution also proceeds somehow differently, the final wave fronts in both cases look quite similar (compare Fig. 8c-e with Fig. 9f-i).

A substantial sensitivity of the model results to the initial wave amplitude is more evident considering an ISW with initial amplitude of $10 \mathrm{~m}$ (see Fig. 10). In this case the Mach stem is also clear (Fig. 10b), but in contrast to the former cases, it does not attach to the leading wave front, neither in the beginning of the experiment, nor at any further moment in time. It seems that the collision of secondary wave with the leading wave will never occur. After $500 \mathrm{~h}$ of evolution there is no substantial decrease of the distance between the leading and second waves (compare panels $\mathrm{d}$ and $\mathrm{e}$ in Fig. 10: the second perturbation at $t=501.4 \mathrm{~h}$ is still very far from the leading wave as it was at $t=90.3 \mathrm{~h}$ ). The secondary Kelvin wave is quite weak having its maximum amplitude of only $2.8 \mathrm{~m}$. Therefore, an important conclusion from this experiment is that the discussed above "compression" of the leading Kelvin waves with formation of a quasi-steady form of the wave packet does not take place in a weakly nonlinear case. Probably there exists an amplitude (energy) threshold above which the energy accumulated by the secondary wave exceeds the energy leakage due to radiation and viscous damping. Above this level, the effect of Kelvin waves packet formation is persistent but below it the energy scattering due to radiation suppresses all other effects.

It is worthwhile mentioning here that smaller solitary waves lose their energy slowlier. This fact is evident in Fig. 11 where the temporal dependence of the normalized energy calculated for the initial 83,31 and $10 \mathrm{~m}$ amplitude ISWs is presented. This result is in line with the conclusion formulated on the basis of the RMKP equation by Akylas (1991), which under an assumption of weak rotation $(R \gg l)$, 


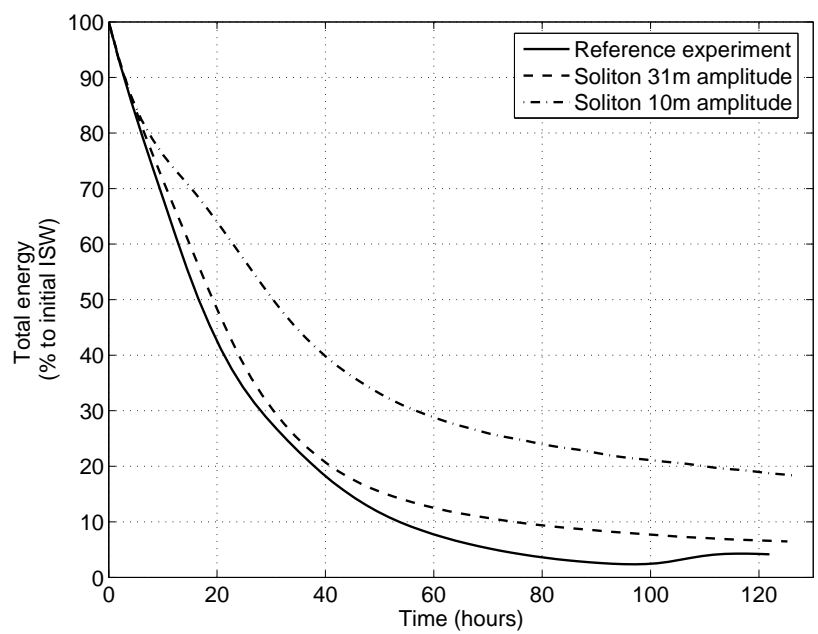

Fig. 11. The total wave energy (kinetic and available potential) of the leading ISW normalized by its initial energy. Solid, dashed and dashed-dotted lines correspond to incoming 83, 31 and $10 \mathrm{~m}$ amplitude ISWs, respectively.

the radiation damping of the waves due to non-linear resonance with Poincaré modes weakens when initial amplitude decreases.

\subsection{Sensitivity to rotation and width of the channel}

The previous sections have shown that rotation is responsible for the wave damping due to the generation of secondary Poincare waves. It is entirely expected that this effect should enhance with the increase of latitude. However, in a very general oceanographic context, not only the Coriolis parameter control the rotational effects. The measure of dependence is defined by the ratio between the Coriolis force and the pressure gradients. That is to say, any possible effect of rotation can be evaluated comparing the internal Rossby radius of deformation with a horizontal scale of the phenomenon. As long as the cross-channel motions are governed by the Coriolis acceleration, a natural horizontal scale in our case should be the width of the channel.

Two additional experiments were conducted with the aim of studying the sensitivity to rotation. In the first one, all the parameters were kept the same as in the RE except for the latitude which was decreased to $36^{\circ}$ (to a value where the Strait of Gibraltar is situated). In the second experiment, the latitude remained the same as in the RE and the width of the channel was two times larger.

An overall efficiency of the generation of radiated secondary waves can be estimated considering the energy loss. Figure 12 shows the temporal variability of the normalized energy of the leading ISW for all cases compared. Provided that the effect of ordinary dissipation due to viscosity is substantially weaker (it was discussed in Sect. 3, see Fig. 5),

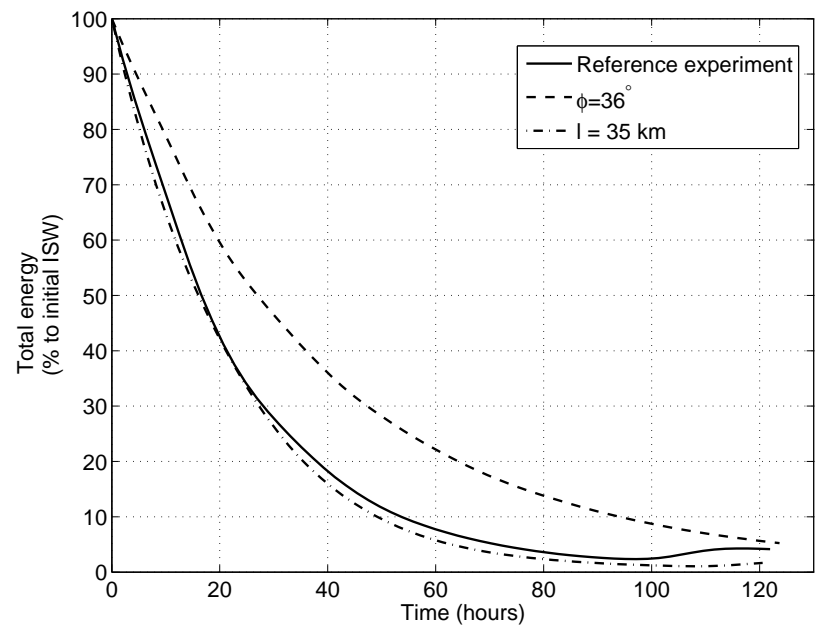

Fig. 12. The total wave energy (kinetic and available potential) of the leading ISW normalized by its initial value. Solid line represents the RE. Dashed line corresponds to the latitude of $\phi=36^{\circ}$, while dotted-dashed line was built for the channel with $l=35 \mathrm{~km}$ (all other parameters were as in the RE).

changes in the energy content can be mainly attributed to the radiation damping.

Figure 12 confirms the assumption that the radiation damping weakens with the decrease of the Coriolis parameter. The sensitivity run for the latitude $\phi=36^{\circ}$ shows that the amount of energy of the leading ISW after $50 \mathrm{~h}$ of evolution is several times larger in the slower rotating system (compare dashed and solid lines in Fig. 12). The implications of a slower radiation damping on the wave evolution can be observed in Fig. 13, showing the plan view of the nearfield of the sensitivity run. The evolution of the wave field is similar to that of the RE but delayed in time, processes proceed slower. For instance, a well-developed secondary Kelvin wave generated from the initial Mach stem can be observed in the RE after $70 \mathrm{~h}$ of evolution (see Fig. 2e), whereas similar structure for the latitude $\phi=36^{\circ}$ appears only after $220 \mathrm{~h}$ (see Fig. 13e). Another example is that after the collision with the leading wave, a quasi-stationary packet of Kelvin waves in the RE is formed after $120 \mathrm{~h}$ (see Fig. $2 \mathrm{~g}$ ) while in this case the transition stage lasts more than $300 \mathrm{~h}$ (see Fig. 13f-i).

In the light of former discussions concerning the effect of rotation, the temporal dependence of the energy of the ISW propagating along the wider channel (dashed-dotted line, Fig. 12) is completely unexpected. It almost perfectly coincides with the wave energy evolution in the RE (solid line, Fig. 12). This fact is in contradiction with the commonly accepted idea that the effect of rotation should become more important when the ratio $l / R$ increases. Note, however, that this is valid whenever $l \cong R$, but for $l \gg R$ an actual value of $l$ does not play any remarkable role. For the configuration and stratification considered here $R=8.3 \mathrm{~km}$, which is about half 

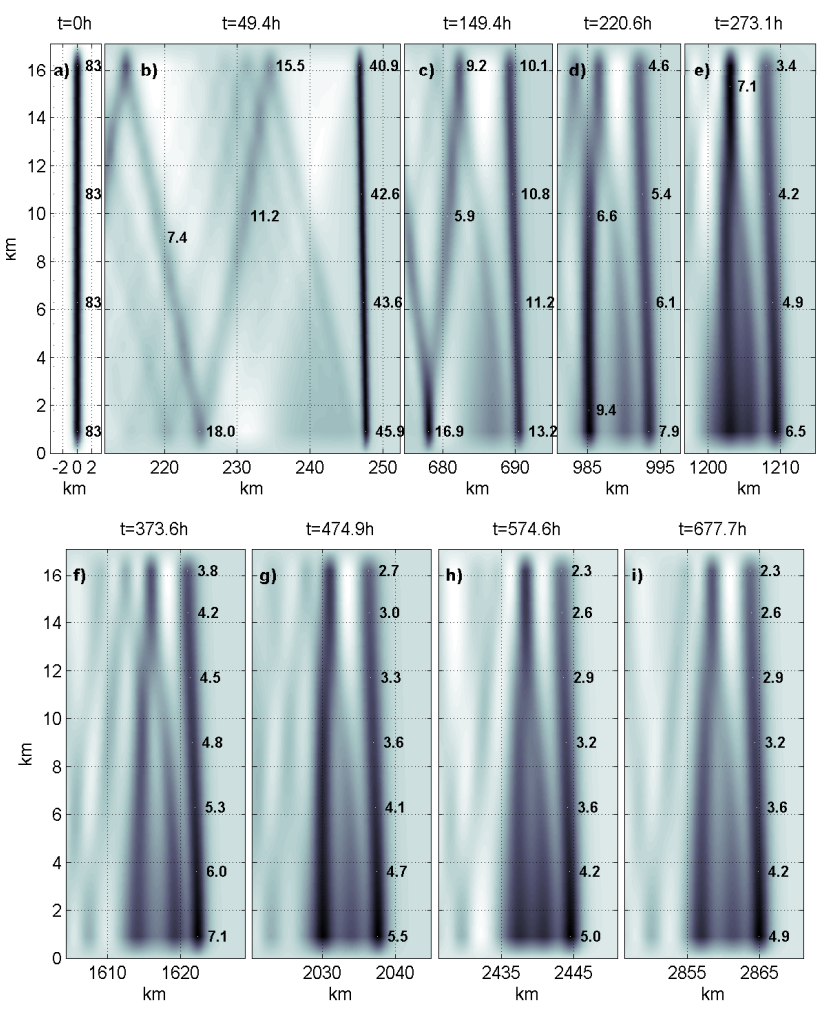

Fig. 13. Same as Fig. 2 but for latitude $36^{\circ}$.

the channel width, and its twofold increase does not affect substantially the integral characteristics of radiated waves.

Another (more quantitative) explanation of this effect can be found in terms of the theory of nonlinear coupling of Kelvin and Poincaré modes. The exchange of energy between these modes takes place because of the coefficients $\left|C_{0 p q}\right|(p+q=1,2, \ldots)$ presented in Eq. (6), which do not depend on all input parameters monotonously. Figure 14 shows the dependence of the coefficients $\left|C_{001}\right|$ on the channel width for the parameters of the present study. The term $C_{001} A_{0}^{2} A_{1}$ is really expected to be the main responsible for the resonance between the modes, it follows from the fact that the first modes dominate as it normally occurs in many physical systems and can be observed in Fig. 6 .

The value of the coefficient $\left|C_{001}\right|$ is almost the same for $l=17$ and $35 \mathrm{~km}$, which is why the integral energy characteristics of two processes are similar. It is important to remark that zero value for $\left|C_{001}\right|$ is not an indicator that the energy exchange between the modes is absent and they propagate independently. In such a situation other coefficients $\left|C_{0 p q}\right|$ become more important in the energy balance. This is well illustrated in Fig. 14, where $\left|C_{002}\right|,\left|C_{003}\right|$ are also shown.

The similarity of an integral energy balance of the two cases discussed above does not mean that all kinematic characteristics of two processes coincide as well. In wider channels the mechanism of reflection, formation of Mach stems

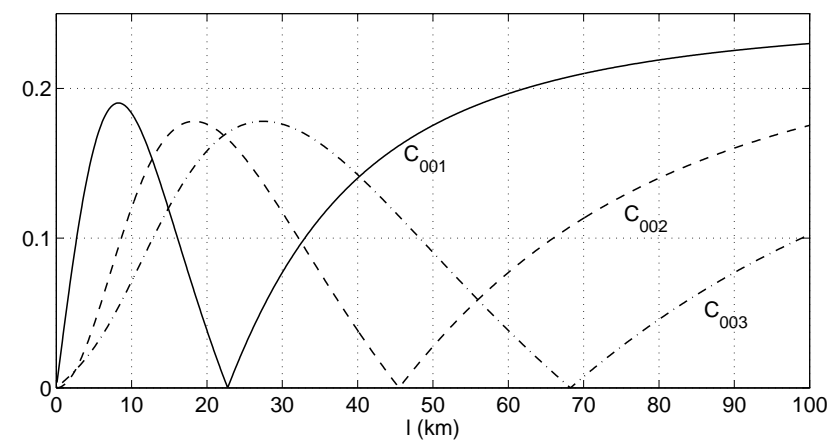

Fig. 14. The dependence of coefficients $\left|C_{001}\right|,\left|C_{002}\right|,\left|C_{003}\right|$ on the width of the channel. The mean value of the propagation speed of the soliton during the first $100 \mathrm{~h}$ of evolution has been taken $(c=$ $\left.1.30 \mathrm{~ms}^{-1}\right)$.

and their subsequent evolution into secondary Kelvin waves looks more pronounced and more efficient (compare Fig. 15 where the wave evolution in the wider channel is presented, and 2). In some aspects the wave evolution in a wider channel proceeds faster. For instance, the second event of secondary Kelvin wave formation in Fig. 15 takes place after $118 \mathrm{~h}$ (see panel f), whereas in the RE it happened only after $200 \mathrm{~h}$ (see Fig. 8b). Another striking feature is the formation of the Mach stems in the middle of the channel (panels e and f) which was less obvious in the RE.

\section{Summary and conclusions}

In this paper a long term evolution of strongly nonlinear solitary waves in a rotating channel is investigated using the fully-nonlinear non-hydrostatic Massachusetts Institute of Technology general circulation model. All numerical experiments are initialized using a two-dimensional internal solitary wave. The process of wave evolution is studied over several hundred hours in order to understand the long-term influence of the rotation. One particular question was to find out what could be an ultimate state of the wave field. An evident distinction of this study from several already completed research (see, for instance, Akylas, 1991, and references herein) is that in our approach we do not apply any restriction on the wave amplitude, i.e. the problem is investigated beyond the applicability of the weakly-nonlinear rotation modified Kadomtsev-Petviashvili (RMKP) equation. The last circumstance allowed us to find several interesting features of wave evolution which are not a direct consequence of the RMKP equation. This remark concerns basically the formation of a slowly attenuated packet of Kelvin waves as an ultimate state of the evolution of a large-amplitude internal solitary wave. At the first stage of evolution, an initially twodimensional ISW starts to evolve according to the classical scenario described by the RMKP equation (e.g., Katsis and Akylas, 1987; Grimshaw and Tang, 1990). More specifically, 

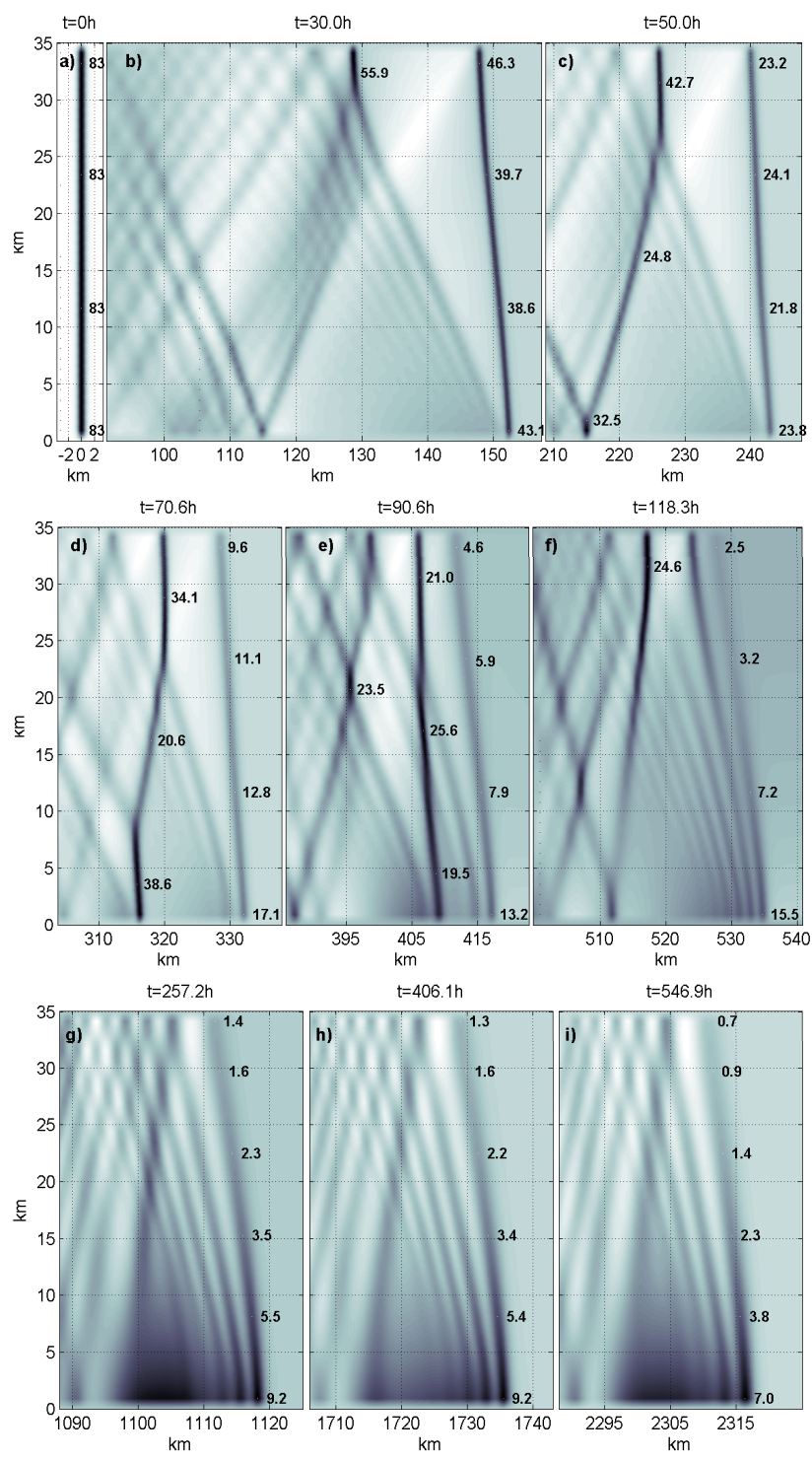

Fig. 15. Same as Fig. 2 but for a channel of $35 \mathrm{~km}$ width.

it starts to transform into a Kelvin wave (with exponential decay of the wave amplitude across the channel) and its front curved backwards. This transition is accompanied by a permanent radiation of secondary Poincaré waves attached to the leading wave.

In a weakly nonlinear theory, such wave radiation inevitably leads to a damping and complete dissipation of the whole wave field. However, as it was found here, the strongly-nonlinear limit of this process allows for an extra intermediate stage of evolution, viz. the formation of a slowly attenuating packet of leading Kelvin waves which is able to propagate for several hundred hours as an allocated deterministic object. The reason for this behaviour can be explained in terms of a permanent reconstruction and modification of the leading wave packet due to the interaction with the secondary wave tail. In fact the leading waves not only lose their energy due to radiation but also recover part of it in the course of interaction with secondary Kelvin waves. The latter are permanently generated behind the leading waves, accumulate the energy which is lost by the leading packet and grow in amplitude. At a final stage they overtake the leading wave returning in exchange some part of the lost energy. This process is discussed in Sect. 3 in greater detail and is illustrated in Figs. 2 and 4.

An important role in this mechanism of wave-wave interaction is played by the Mach stems permanently generated in the near-field due to the interaction of obliquely propagating radiated waves reflected from the walls (they can be identified in Figs. 2, 8, 9, 13, 15). These fragments work as points of energy condensation. The Mach stems absorb energy from the leading wave eventually transforming into a secondary Kelvin wave. Normally, they are more energetic than the leading one, which is why they start to overtake the latter returning part of energy. This process is sensitive to the initial wave amplitude, rotation and channel width.

It is interesting that not all the initial waves were able to follow the described mechanism of transformation from solitary wave into a slowly decaying packet of Kelvin waves. The numerical experiments clearly demonstrated that there is an amplitude threshold below which the discussed scenario does not take place. In our case it was the incoming 10-m amplitude ISW which just attenuated after $500 \mathrm{~h}$ of evolution without formation of the leading packet. This is probably the reason why the result obtained here on the formation of the packets of Kelvin waves was not reported in some other similar works based on the RMKP equation. However, this conclusion is similar to that formulated by Helfrich (2007) and Grimshaw and Helfrich (2008), who found (but for infinite rotating basins) that the formation of packets of ISWs from the initial ISW is possible if the initial accumulated energy is large enough.

Acknowledgements. This work was supported by the UK NERC grant NE/F010214/1. JCSG acknowledges a post-graduate fellowship from Consejería de Innovación Ciencia y Empresa, Junta de Andalucía, Spain (research project RNM 968). We are thankful to Dr. García-Lafuente and Dr. Stashchuk for their comments and geat interest on the paper. The authors also thankfully acknowledge the computer resources and technical support provided by the SCBI (Supercomputing and Bioinformatics) center of the University of Malaga.

Edited by: E. Garcia Ladona

Reviewed by: two anonymous referees

\section{References}

Akylas, T. R.: On the radiation damping of a solitary wave in a rotating channel, in: Mathematical Approaches in Hydrodynamics, edited by: Miloh, T., SIAM, Philadelphia, 175-181, 1991.

Briscoe, M.: Tides, solitons and nutrients, Nature, 312, 1-5, 1984. 
Garrett, C.: Internal tides and ocean mixing, Science, 301, 18581859, 2003.

Gerkema, T.: A unified model for the generation and fission of internal tides in a rotating ocean, J. Mar. Res., 54, 421-450, 1996.

Gerkema, T. and Zimmerman, J. T. F.: Generation of nonlinear internal tides and solitary waves, J. Phys. Oceanogr., 25, 10811094, 1995.

Gilman, O. A., Grimshaw, R., and Stepanyants, Y. A.: Dynamics of internal solitary waves in a rotating fluid, Dyn. Atmos. Oceans, 23, 403-411, 1996.

Grimshaw, R.: Evolution equations for weakly nonlinear, long internal waves in a rotating fluid, Stud. Appl. Math., 73, 1-33, 1985.

Grimshaw, R., He, J.-M., and Ostrovsky, L.: Terminal damping of a solitary wave due to radiation in rotational system, Stud. Appl. Math., 101, 197-210, 1998.

Grimshaw, R. and Helfrich, K. R.: Long-time solutions in the Ostrovsky equation, Stud. Appl. Math., 121, 71-78, 2008.

Grimshaw, R. and Melville, W. K.: On the derivation of the modified Kadomtsev-Petviashvili equation, Stud. Appl. Math., 80, 183-202, 1988.

Grimshaw, R. and Tang, S.: The rotation-modified KadomtsevPetviashvili equation: an analytical and numerical study, Stud. Appl. Math., 83, 223-248, 1990.

Helfrich, K. R.: Decay and return of internal solitary waves with rotation, Phys. Fluids, 19, 026601, 2007.

Helfrich, K. R. and Grimshaw, R. H. J.: Nonlinear disintegration of the internal tide, J. Phys. Oceanogr., 38, 686-701, 2008.

Katsis, C. and Akylas, T. R.: Solitary internal waves in a rotating channel: A numerical study, Phys. Fluids, 30, 297-301, 1987.

Leonov, A. I.: The effect of the earth's rotation on the propagation of weakly-nonlinear surface and internal long waves, Annals NY Acad. Sci., 373, 150-159, 1981.
Maxworthy, T.: Experiments on internal solitary waves, J. Fluid Mech., 129, 365-383, 1983.

Marshall, J., Adcroft, A., Hill, C., Perelman, L., and Heisey, C.: A finite-volume, incompressible Navier-Stokes model for studies of the ocean on parallel computers, J. Geophys. Res., 102, 57335752, 1997.

Melville, W. K., Tomasson, G. G., and Renouard, D. P.: On the stability of Kelvin waves, J. Fluid Mech., 206, 1-23, 1989.

Munk, W. H. and Wunsch, C.: Abyssal recipe II: Energetics of tidal and wind mixing, Deep-Sea Res. I, 45, 1977-2010, 1998.

Ostrovsky, L. A.: Nonlinear internal waves in a rotating ocean, Oceanology, 18, 181-191, 1978.

Renouard ,D. P., Chabert dHieres, G., and Zhang, X.: An experimental study of strongly nonlinear waves in a rotating system, J. Fluid Mech., 177, 381-394, 1987.

Vlasenko, V., Stashchuk, N., and Hutter, K: Baroclinic tides: theoretical modeling and observational evidence, Cambridge University Press, 2005.

Vlasenko, V., Sánchez Garrido, J. C., Stashchuk, N., García Lafuente, J., and Losada, M.: Three Dimensional Evolution of large-amplitude internal waves in the Strait of Gibraltar, J. Phys. Oceanogr., 39, 2230-2246, 2009.

Wesson, J. and Gregg, M.: Turbulent dissipation in the Strait of Gibraltar and associated mixing, in: Small-Scale Turbulence and Mixing in the Ocean, Elsevier Sci., Nihoul-Jamart, New-York, 201-212, 1988.

Yang, Y. J., Tang, T. Y., Chang, M. H, Liu, A. K, Hsu, M.-K., and Ramp, S. R: Solitons northeast of Tung-Sha Island during the ASIAEX pilot studies, IEEE J. Oceanic Eng., 29, 1182-1199, 2004. 